\title{
Data-driven Controller Tuning Based on a Frequency Criterion
}

\author{
Daniel Garcia \\ Alireza Karimi \\ Roland Longchamp \\ Laboratoire d'Automatique, École Polytechnique Fédérale de Lausanne (EPFL), \\ CH-1015 Lausanne, Switzerland. e-mail: daniel.garcia@epfl.ch
}

\begin{abstract}
An iterative controller auto-tuning method based on a frequency criterion is proposed. The frequency criterion is defined as the weighted sum of squared errors between the desired and measured gain margin, phase margin and crossover frequency. A relay feedback test is used to automatically obtain a non-parametric model of the open-loop system in a very important region for control design. The gain and phase margins as well as the crossover frequency are estimated with the non-parametric model using interpolations. The gradient and Hessian of the frequency criterion can be expressed in terms of the derivatives of the open-loop system with respect to the frequency. These derivatives can also be estimated with a good accuracy thanks to the non-parametric model. Since no assumptions are made on the plant, the method is valid for a very wide class of linear systems. Simulation examples illustrate the effectiveness and simplicity of the proposed method.
\end{abstract}

\section{INTRODUCTION}

Simple controllers such as the conventional PID controllers are still widely used in practical applications. In spite of their very simple structure, they often perform well and meet the specifications, provided that their parameters are properly chosen. Therefore, several different automatic tuning procedures with varying objectives and complexity are desirable. Many methods are already available, but they are derived for particular processes and situations, and consequently they apply well only to their own area. It is hence desirable to propose simple and fast controller tuning methods that are not restricted to a controller structure and that achieve high performances for a very wide class of linear processes.

The available methods are normally based on a first [1] or a second-order [9] model with dead time obtained from the measurement of one or more points on the frequency response of the process. The problem with the first-order plus dead time models is that they are absolutely not representative of the majority of real plants. Peaks in the frequency response cannot, among others, be generated by such models. One can expect that the methods based on first-order models applied to real plants will not provide the desired results. Second-order plus dead time models approximate real systems much better [9]. However the identification procedure, which consists of solving iteratively non-linear trigonometric equations, may fail. Additionally, the measured points are usually obtained with the describing function method applied to a relay feedback test [2]. This method is approximative and poor results may be obtained for some systems, like processes with a long time-delay and those having a low relative degree.

In recent years, considerable attention has been given to data-driven controller tuning without or with little use of models. These tuning procedures use directly the experimental closed-loop data and do not suffer from unmodeled dynamics. An iterative controller tuning procedure based on the minimization of a frequency criterion has been proposed in [5], [4]. This method shows promising results but is restricted to stable minimum phase systems.

The main contribution of this paper is to extend the validity of the iterative tuning procedure in [5] to a very large class of linear systems, including oscillatory, non-minimum phase and unstable processes. The frequency criterion is defined as the weighted sum of squared errors between the desired and measured gain margin, phase margin and crossover frequency. The gradient and Hessian of the criterion can be expressed with the derivatives of the amplitude and phase of the system with respect to the frequency at the crossover frequency (the frequency at which the amplitude is one) and at the ultimate frequency (the frequency at which the phase is $-\pi)$. It is shown that, with the only knowledge of multiple estimated points of the process open-loop frequency response, obtained at each iteration with only one experiment, not only the phase margin, the gain margin and the crossover frequency can be approximated with high precision but also the gradient and Hessian of the frequency criterion. The advantage of the proposed method is that no assumption is made on the process and the controller structure. The only requirement is that a stabilizing controller exists prior to the tuning procedure, and the purpose of the proposed method is to improve the performance, stability and robustness of the closed-loop system. Simulation results show the efficiency and the fast convergence of the proposed method.

The paper is organized as follows: A method, based on a closed-loop relay experiment, that automatically estimates multiple points of the open-loop frequency response, is presented in Section II. Section III shows how the robustness margins, the crossover frequency as well as the derivatives of the open-loop frequency response with respect to the frequency can be approximated with such a non-parametric model. The iterative tuning method is then presented in Section IV and simulation examples are provided in Section V. Finally some concluding remarks are offered in Section VI. 


\section{Multiple Points Measurement Using A Closed-LoOp RELAY EXPERIMENT}

The proposed measurement method is derived from the closed-loop relay feedback test proposed in [10] to generate a limit cycle at the crossover frequency of the open-loop transfer function and in [7] to measure the phase margin using the describing function approach. The accuracy of the method proposed in [7] is however poor: Experiments have shown relative errors between the measurements and the real values of the crossover frequencies of about $40 \%$ [7]. Errors come from the fact that the transfer function seen by the relay has a relative degree of 1 , and thus the describing function assumption is not guaranteed.

The method proposed in this section will benefit from the properties of the closed-loop relay experiment to obtain a non-parametric model with a good precision using the Fourier analysis. On the one hand the experiment automatically provides excitations to the system in the desired frequency region. On the other hand the low relative degree of the transfer function seen by the relay makes it possible to measure other points of the open loop frequency response at frequencies corresponding to higher odd harmonics. However, in order to additionally provide excitation to the process at different others frequencies, two simple modifications are made to the scheme.

The modified closed-loop relay experiment is shown in Fig. 1. The first modification consists in replacing the symmetric relay by a biased relay. A biased relay is simply obtained by adding a constant value $\mu$ at the output of a symmetric relay. $\mu$ should be chosen smaller than $d$, where $d$ is the output amplitude of the standard (symmetric) relay (see Fig. 1). This change adds the even harmonics with the frequencies $0,2 \omega_{0}, \ldots$ at the relay output, where $\omega_{0}$ is the fundamental frequency of the signal. Thus, further excitation frequencies are supplied at the closed-loop system.

The second change is to superimpose a parasitic relay to the standard test. The parasitic relay has been introduced in [8] and is defined as:

$$
\begin{cases}u_{r_{2}}(0)=\alpha d & \\ u_{r_{2}}(k)=-\alpha d \operatorname{sign}\left(u_{r_{2}}(k-1)\right), & \text { if } u_{r_{1}}(k-1)>0 \text { and } u_{r_{1}}(k)<0 \\ u_{r_{2}}(k)=u_{r_{2}}(k-1), & \text { otherwise }\end{cases}
$$

where $u_{r_{1}}(k)$ and $u_{r_{2}}(k)$ are the sampled output of the signals $u_{r_{1}}(t)$ and $u_{r_{2}}(t)$ respectively (see Fig. 1) and $\alpha$ is a constant coefficient which is recommended to choose between 0.1 and 0.3 [8]. The period of the output of the parasitic relay is twice as large as the period of the output of the standard relay. Thus, the excitations at $0.5 \omega_{0}, 1.5 \omega_{0}$, $2.5 \omega_{0}, \ldots$ are now available, and the fundamental frequency $\omega_{0}$ is closed to the crossover frequency $\omega_{c}$. A non-parametric model of the process frequency response can now be obtained from signals resulting from the proposed experiment as follows.

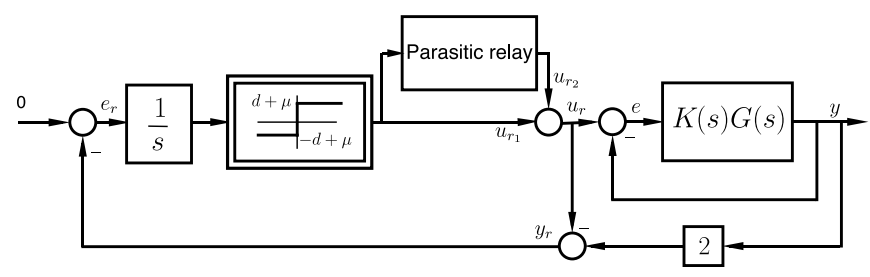

Fig. 1. Modified Relay experiment for multiple points measurements

Let $u_{r}(t)$ be the input of the closed-loop system and $y_{r}(t)$ be its output (see Fig. 1). Define:

$$
F(s)=\frac{Y_{r}(s)}{U_{r}(s)}=\frac{K(s) G(s)-1}{K(s) G(s)+1}
$$

The signals which are used for the identification are $u_{r}(t)$ and $y_{r}(t)$, because $u_{r}(t)$ is not affected by noise and on the other hand the transfer function $F(s)$ does not damp the higher harmonics, thanks to its relative degree 0. Consider $N_{s}$ sampled values $u_{r}\left(k T_{s}\right)$ and $y_{r}\left(k T_{s}\right), 1<k<N_{s}$ with the sampling period $T_{s}$, of the signals $u_{r}(t)$ and $y_{r}(t)$. The periodogram of these sequences are defined respectively as [6]:

$$
\begin{aligned}
U_{r}(\omega) & =\frac{1}{\sqrt{N_{s}}} \sum_{k=1}^{N_{s}} u_{r}\left(k T_{s}\right) e^{-j \omega k T_{s}} \\
Y_{r}(\omega) & =\frac{1}{\sqrt{N_{s}}} \sum_{k=1}^{N_{s}} y_{r}\left(k T_{s}\right) e^{-j \omega k T_{s}}
\end{aligned}
$$

If $N_{s} T_{s}=p T$, where $p$ is any positive integer and $T$ the period of the signals $u_{r}(t)$ and $y_{r}(t)$, then the estimated discrete transfer function:

$$
F\left(e^{j \omega}\right)=\frac{Y_{r}(\omega)}{U_{r}(\omega)}
$$

satisfies:

- $F\left(e^{j \omega}\right)$ is defined only for a fixed number of frequencies (those where $U_{r}(\omega) \neq 0$ )

- At these frequencies the estimates are unbiased and its variance due to disturbances decays with $\frac{1}{p}$

These results are demonstrated in [6] and can be used to estimate the continuous transfer function frequency response $F(j \omega)$ at the frequencies $\omega_{i}$ that are contained in the excitation signals, provided that the sampling period is properly chosen. The open-loop frequency response can then be estimated at $\omega_{i}(i=1, \ldots)$ as:

$$
K\left(j \omega_{i}\right) G\left(j \omega_{i}\right)=\frac{F\left(j \omega_{i}\right)+1}{1-F\left(j \omega_{i}\right)}
$$

\section{RoBUStness MARgINS AND DERIVATIVES ESTIMATIONS}

\section{A. Computation of the Estimates}

Consider a non-parametric model of the open-loop transfer function which consists of a finite set of points $L_{i}$ of its frequency response in the ascending order of the corresponding 
frequency:

$$
L_{i}=K\left(j \omega_{i}\right) G\left(j \omega_{i}\right), \quad i=1, \ldots, N, \quad \omega_{i-1}<\omega_{i}
$$

Suppose also that the crossover frequency $\omega_{c}$ and the ultimate frequency $\omega_{u}$ are located between $\omega_{1}$ and $\omega_{N}$. Such a nonparametric model can be obtained by the method proposed in Section II. Based on this model, the objective is to estimate the crossover and the ultimate frequency, the gain and phase margins, as well as the derivatives of the amplitude and phase of the open-loop frequency response with respect to $\omega$ at $\omega_{c}$ and $\omega_{u}$ :

$$
\begin{aligned}
& s_{a_{c}}=\left.\frac{d \log |K(j \omega) G(j \omega)|}{d \log \omega}\right|_{\omega_{c}} \quad s_{p_{c}}=\left.\frac{d \angle(K(j \omega) G(j \omega))}{d \log \omega}\right|_{\omega_{c}} \\
& s_{a_{u}}=\left.\frac{d \log |K(j \omega) G(j \omega)|}{d \log \omega}\right|_{\omega_{u}} \quad s_{p_{u}}=\left.\frac{d \angle(K(j \omega) G(j \omega))}{d \log \omega}\right|_{\omega_{u}}
\end{aligned}
$$

These derivatives will then be used in the controller tuning procedure to compute the gradient and Hessian of a frequency criterion that will be minimized iteratively.

The crossover and ultimate frequencies, as well as the phase and gain margins, are obtained by linear interpolations. The linear interpolation function is defined as follows. Let $y=f(x)$ be a continuous function between $x=x_{1}$ and $x=x_{2}, x_{1}<x_{2}$. The interpolation function, which is used to find the value $y_{I}$ of the function $f$ at $x_{I}$, where $x_{1}<x_{I}<x_{2}$, is defined as :

$$
y_{I}=\operatorname{Interp}\left(x_{1}, x_{2}, y_{1}, y_{2}, x_{I}\right)=y_{1}+\left(x_{I}-x_{1}\right) \frac{y_{2}-y_{1}}{x_{2}-x_{1}}
$$

where $y_{1}=f\left(x_{1}\right)$ and $y_{2}=f\left(x_{2}\right)$.

Since the amplitude and phase of a system on the Bode diagram are almost piecewise linear, the linear interpolations are made on logarithmic scales for frequency and amplitude.

The results of the interpolation gives the approximated crossover frequency :

$$
\log \omega_{c}=\operatorname{Interp}\left(\log \left|L_{n}\right|, \log \left|L_{n+1}\right|, \log \omega_{n}, \log \omega_{n+1}, 0\right)
$$

where $L_{n}$ and $L_{n+1}$ are the two points of the non-parametric model such that $\left|L_{n}\right|>1>\left|L_{n+1}\right|, \omega_{n}$ and $\omega_{n+1}$ are the corresponding frequencies, respectively. The phase margin is approximated as:

$$
\Phi_{m}=\pi+\operatorname{Interp}\left(\log \omega_{n}, \log \omega_{n+1}, \angle L_{n}, \angle L_{n+1}, \log \omega_{c}\right)
$$

In a similar way, the ultimate frequency is given by:

$$
\log \omega_{u}=\operatorname{Interp}\left(\angle L_{m}, \angle L_{m+1}, \log \omega_{m}, \log \omega_{m+1},-\pi\right)
$$

where $L_{m}$ and $L_{m+1}$ are the two points of the non-parametric model, such that $\angle L_{m}>-\pi>\angle L_{m+1}, \omega_{m}$ and $\omega_{m+1}$ are the corresponding frequencies, respectively. The absolute value of the ultimate point $K_{u}$, which is the inverse of the gain margin is computed as:

$$
K_{u}=10^{\text {Interp }\left(\log \omega_{m}, \log \omega_{m+1}, \log \left|L_{m}\right|, \log \left|L_{m+1}\right|, \log \omega_{u}\right)}
$$

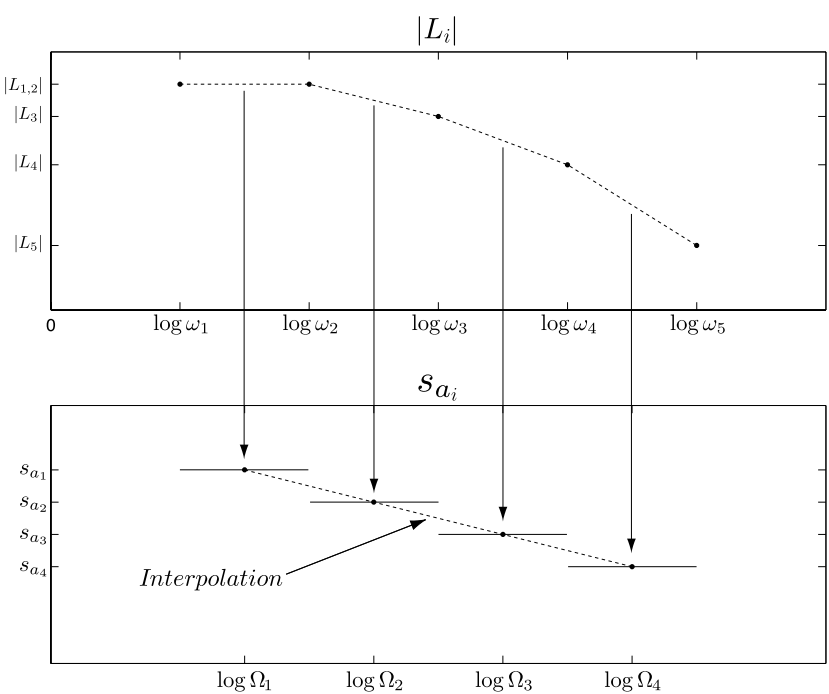

Fig. 2. Estimation of the slopes of amplitude

Now it only remains to approximate the slope of amplitude and phase of the open-loop frequency response with respect to $\omega$ at $\omega_{c}$ and $\omega_{u}$. For this purpose we define for every frequency interval $\left[\omega_{i}, \omega_{i+1}\right]$ an averaged slope of amplitude and phase:

$$
s_{a_{i}}=\frac{\log \left|L_{i+1}\right|-\log \left|L_{i}\right|}{\log \omega_{i+1}-\log \omega_{i}}, \quad s_{p_{i}}=\frac{\angle L_{i+1}-\angle L_{i}}{\log \omega_{i+1}-\log \omega_{i}}
$$

Extensive simulations on different systems have shown that the slopes $s_{a_{i}}$ and $s_{p_{i}}$ constitute good approximations for the frequency which is at the middle of the interval $\left[\omega_{i}, \omega_{i+1}\right]$ on a logarithmic scale:

$$
\Omega_{i}=10^{\frac{\log \omega_{i}+\log \omega_{i+1}}{2}}=10^{\frac{\log \left(\omega_{i} \omega_{i+1}\right)}{2}}
$$

Thus we define the slopes $s_{a_{i}}$ and $s_{p_{i}}$ as being the slopes at the frequency $\Omega_{i}$ (see Fig. 2). Again, the slopes at the crossover and ultimate frequencies are obtained by linear interpolation:

$$
\begin{aligned}
s_{a_{c}} & =\left.\frac{d \log |K(j \omega) G(j \omega)|}{d \log \omega}\right|_{\omega_{c}} \\
& \approx \operatorname{Interp}\left(\log \Omega_{n}, \log \Omega_{n+1}, s_{a_{n}}, s_{a_{n+1}}, \log \omega_{c}\right) \\
s_{p_{c}} & =\left.\frac{d \angle(K(j \omega) G(j \omega))}{d \log (\omega)}\right|_{\omega_{c}} \\
& \approx \operatorname{Interp}\left(\log \Omega_{n}, \log \Omega_{n+1}, s_{p_{n}}, s_{p_{n+1}}, \log \omega_{c}\right) \\
s_{a_{u}} & =\left.\frac{d \log |K(j \omega) G(j \omega)|}{d \log (\omega)}\right|_{\omega_{u}} \\
& \approx \operatorname{Interp}\left(\log \Omega_{m}, \log \Omega_{m+1}, s_{a_{m}}, s_{a_{m+1}}, \log \omega_{u}\right) \\
s_{p_{u}} & =\left.\frac{d \angle(K(j \omega) G(j \omega))}{d \log (\omega)}\right|_{\omega_{u}} \\
& \approx \operatorname{Interp}\left(\log \Omega_{m}, \log \Omega_{m+1}, s_{p_{m}}, s_{p_{m+1}}, \log \omega_{u}\right)
\end{aligned}
$$

where $n$ is the index for which $\Omega_{n}<\omega_{c}<\Omega_{n+1}$, and $m$ the index for which $\Omega_{m}<\omega_{u}<\Omega_{m+1}$. 


\section{B. Precision of the Estimates}

Precision of the estimates of the phase margin, gain margin, crossover frequency as well as the loop derivatives of amplitude and phase depends on the system dynamics and the accuracy of the non-parametric identification.

If the model is accurate, the estimates are practically exact. Simulations have shown that the relative errors are smaller than $1 \%$ for typical models of industrial plants. The estimations of the robustness margins are much more accurate than those obtained by standard relay methods using the describing function analysis. Moreover, all the estimates are obtained with only one experiment.

In a realistic environment there are however identification errors, that come from the disturbances and noise. It should be noted that methods based on Fourier transform are unaffected by step-like load disturbance, which is a common case in practice. If the experiment of Section II is used for the points estimation in the presence of significant measurement noise, the use of an hysteresis in the relay is a simple way to reduce its influence. Moreover, the use of several periods for the identification reduces the effect of the noise on the estimates (see Section II). In a very noisy environment, conventional non-parametric frequency-domain methods should be preferred to the proposed one, because the amplitude of each harmonic can be chosen independently. Larger amplitudes for the excitation at the higher harmonics can thus be chosen.

\section{Iterative Procedure for Controller Tuning}

First of all, a performance criterion in the frequency domain is defined as follows:

$$
\begin{aligned}
J(\rho)=\frac{1}{2}\left(\lambda_{1}\left(\omega_{c}-\omega_{d}\right)^{2}+\lambda_{2}\left(\Phi_{m}-\right.\right. & \left.\Phi_{d}\right)^{2} \\
& \left.+\lambda_{3}\left(K_{u}-K_{d}\right)^{2}\right)
\end{aligned}
$$

where $\rho$ is the vector of the controller parameters of dimension $n_{\rho}, \lambda_{1}, \lambda_{2}$ and $\lambda_{3}$ are weighting factors, $\omega_{c}$ and $\omega_{d}$ are respectively the measured and desired crossover frequencies, $\Phi_{m}$ and $\Phi_{d}$ are the measured and desired phase margins and $K_{u}=\left|L\left(j \omega_{u}\right)\right|$ and $K_{d}$ are respectively the measured and desired inverse of the gain margins. Such a performance criterion in the frequency domain has been introduced in [5]. Then the controller parameters minimizing the criterion can be obtained iteratively by the Gauss-Newton method:

$$
\rho_{i+1}=\rho_{i}-\gamma_{i} R^{-1} J^{\prime}\left(\rho_{i}\right)
$$

where $i$ is the iteration number, $\gamma_{i}$ is the step size, $R$ is a positive definite matrix of dimension $n_{\rho} \times n_{\rho}$ that can be chosen equal to the Hessian $H$ for a fast convergence and $J^{\prime}(\rho)$ is the gradient of the criterion with respect to $\rho$.

The gradient of the criterion is given by:

$$
\begin{aligned}
J^{\prime}(\rho)=\lambda_{1}\left(\omega_{c}-\omega_{d}\right) \frac{\partial \omega_{c}}{\partial \rho}+ & \lambda_{2}\left(\Phi_{m}-\Phi_{d}\right) \Phi_{m}^{\prime} \\
& +\lambda_{3}\left(K_{u}-K_{d}\right) K_{u}^{\prime}
\end{aligned}
$$

where $\Phi_{m}^{\prime}$ is the derivative of the phase margin with respect to $\rho$ and $K_{u}^{\prime}$ is the derivative of the ultimate loop gain with respect to $\rho$.

$\Phi_{m}^{\prime}$ is computed through the chain rule as follows:

$$
\Phi_{m}^{\prime}=\frac{\partial \Phi_{m}}{\partial \rho}+\left.\frac{\partial \Phi_{m}}{\partial \omega}\right|_{\omega_{c}} \frac{\partial \omega_{c}}{\partial \rho}
$$

Now replacing $\Phi_{m}$ in the above equation by $\angle L\left(j \omega_{c}\right)+\pi$ gives:

$$
\Phi_{m}^{\prime}=\frac{\partial \angle L\left(j \omega_{c}\right)}{\partial \rho}+\left.\frac{\partial \angle L(j \omega)}{\partial \omega}\right|_{\omega_{c}} \frac{\partial \omega_{c}}{\partial \rho}
$$

The first term in the above equation is equal to $\partial \angle K\left(j \omega_{c}\right) / \partial \rho$ which is completely known at each iteration. Furthermore one has:

$$
\left.\frac{\partial \angle L(j \omega)}{\partial \omega}\right|_{\omega_{c}}=\left.\frac{1}{\omega_{c} \ln (10)} \frac{\partial \angle L(j \omega)}{\partial \log \omega}\right|_{\omega_{c}} \approx \frac{s_{p_{c}}}{\omega_{c} \ln (10)}
$$

To compute $\frac{\partial \omega_{c}}{\partial \rho}$, we use the fact that the loop gain at $\omega_{c}$ is by definition always equal to 1 . Its derivative (or the derivative of its logarithm) with respect to $\rho$ is therefore zero:

$$
\frac{\partial \ln \left|L\left(j \omega_{c}\right)\right|}{\partial \rho}+\left.\frac{\partial \ln |L(j \omega)|}{\partial \omega}\right|_{\omega_{c}} \frac{\partial \omega_{c}}{\partial \rho}=0
$$

The first term in the above equation is equal to $\partial \ln \left|K\left(j \omega_{c}\right)\right| / \partial \rho$ which can be easily computed at each iteration. The second term can be approximated as follows:

$$
\begin{aligned}
\left.\frac{\partial \ln |L(j \omega)|}{\partial \omega}\right|_{\omega_{c}} & =\left.\frac{1}{\omega_{c}} \frac{\partial \ln |L(j \omega)|}{\partial \ln \omega}\right|_{\omega_{c}} \\
& =\left.\frac{1}{\omega_{c}} \frac{\partial \log |L(j \omega)|}{\partial \log \omega}\right|_{\omega_{c}} \approx \frac{s_{a_{c}}}{\omega_{c}}
\end{aligned}
$$

Thus $\partial \omega_{c} / \partial \rho$ can be approximated as follows:

$$
\frac{\partial \omega_{c}}{\partial \rho} \approx-\frac{\partial \ln \left|K\left(j \omega_{c}\right)\right|}{\partial \rho} \frac{\omega_{c}}{s_{a_{c}}}
$$

Now it only remains to determine $K_{u}^{\prime}$. This one can be computed through the chain rule as:

$$
K_{u}^{\prime}=\frac{\partial\left|L\left(j \omega_{u}\right)\right|}{\partial \rho}+\left.\frac{\partial|L(j \omega)|}{\partial \omega}\right|_{\omega_{u}} \frac{\partial \omega_{u}}{\partial \rho}
$$

The first term is equal to

$$
\left|G\left(j \omega_{u}\right)\right| \frac{\partial\left|K\left(j \omega_{u}\right)\right|}{\partial \rho}=K_{u} \frac{\partial \ln \left|K\left(j \omega_{u}\right)\right|}{\partial \rho}
$$

which is known at each iteration. The second term can be approximated as follows:

$$
\begin{aligned}
\left.\frac{\partial|L(j \omega)|}{\partial \omega}\right|_{\omega_{u}} & =\left.\frac{\left|L\left(j \omega_{u}\right)\right|}{\omega_{u}} \frac{\partial \ln |L(j \omega)|}{\partial \ln \omega}\right|_{\omega_{u}} \\
& =\left.\frac{\left|L\left(j \omega_{u}\right)\right|}{\omega_{u}} \frac{\partial \log |L(j \omega)|}{\partial \log \omega}\right|_{\omega_{u}} \\
& =\frac{\left|L\left(j \omega_{u}\right)\right|}{\omega_{u}} s_{a_{u}}
\end{aligned}
$$


Then $\partial \omega_{u} / \partial \rho$ is computed using the fact that by definition $\angle L\left(j \omega_{u}\right)=-\pi$ and consequently its derivative with respect to $\rho$ is equal to zero, which gives:

$$
\frac{\partial \angle L\left(j \omega_{u}\right)}{\partial \rho}+\left.\frac{\partial \angle L(j \omega)}{\partial \omega}\right|_{\omega_{u}} \frac{\partial \omega_{u}}{\partial \rho}=0
$$

The first term in the above equation is equal to $\partial \angle K\left(j \omega_{u}\right) / \partial \rho$ which again can be computed analytically. The second term can be approximated as follows:

$$
\left.\frac{\partial \angle L(j \omega)}{\partial \omega}\right|_{\omega_{u}} \approx \frac{s_{p_{u}}}{\omega_{u} \ln (10)}
$$

Thus $\partial \omega_{u} / \partial \rho$ can be approximated as follows:

$$
\frac{\partial \omega_{u}}{\partial \rho} \approx-\frac{\omega_{u} \ln (10)}{s_{p_{u}}} \frac{\partial \angle K\left(j \omega_{u}\right)}{\partial \rho}
$$

In the same way, the Hessian of the criterion can be approximated as follows:

$$
\begin{aligned}
H=\lambda_{1} & \frac{\partial \omega_{c}}{\partial \rho}\left(\frac{\partial \omega_{c}}{\partial \rho}\right)^{T}+\lambda_{2} \Phi_{m}^{\prime}\left(\Phi_{m}^{\prime}\right)^{T} \\
& +\lambda_{3} K_{u}^{\prime}\left(K_{u}^{\prime}\right)^{T}+\lambda_{1}\left(\omega_{c}-\omega_{d}\right) \frac{\partial^{2} \omega_{c}}{\partial \rho^{2}} \\
& +\lambda_{2}\left(\Phi_{m}-\Phi_{d}\right) \Phi_{m}^{\prime \prime}+\lambda_{3}\left(K_{u}-K_{d}\right) K_{u}^{\prime \prime}
\end{aligned}
$$

The last three terms can be neglected because they are small especially in the neighborhood of the final solution. In addition, this simplification makes the Hessian always positive which fixes the numerical problems normally encountered in the iterative Newton algorithm. The use of the Hessian matrix in the iterative formula of Eq. (9), instead of $R$, significantly improves the convergence speed.

$$
\begin{aligned}
R=H \approx \lambda_{1} \frac{\partial \omega_{c}}{\partial \rho}\left(\frac{\partial \omega_{c}}{\partial \rho}\right)^{T}+\lambda_{2} \Phi_{m}^{\prime}( & \left.\Phi_{m}^{\prime}\right)^{T} \\
& +\lambda_{3} K_{u}^{\prime}\left(K_{u}^{\prime}\right)^{T}
\end{aligned}
$$

Remark: If the dimension $n_{\rho}$ of the controller parameters is equal to the number of specifications, the iterative solution of Eq. (9) becomes easier to implement. This is the case for example with a PID controller and the criterion of Eq. (8) or with a PI controller and specifications on only phase margin and crossover frequency. Consider without loss of generality the case where $n_{\rho}=3$ with the same number of specifications (on phase margin, gain margin and crossover frequency). The criterion can be written as:

$$
J=\frac{1}{2} Q^{T}(\rho) \Lambda Q(\rho)
$$

where $Q(\rho)$ is the vector of dimension $n_{\rho}$ that contains the differences between measured and desired properties and $\Lambda$ a matrix of dimension $n_{\rho} \times n_{\rho}$ with the $\lambda_{i}, i=1, \ldots, n_{\rho}$ on its diagonal:

$$
Q(\rho)=\left(\begin{array}{c}
\omega_{c}-\omega_{d} \\
\Phi_{m}-\Phi_{d} \\
K_{u}-K_{d}
\end{array}\right) \quad \Lambda=\left(\begin{array}{ccc}
\lambda_{1} & 0 & 0 \\
0 & \lambda_{2} & 0 \\
0 & 0 & \lambda_{3}
\end{array}\right)
$$

The gradient of the criterion $J^{\prime}$ can then be computed as follows:

$$
\begin{aligned}
J^{\prime} & =\frac{1}{2}\left(\frac{\partial Q^{T}(\rho)}{\partial \rho} \Lambda Q(\rho)+\left(Q^{T}(\rho) \Lambda \frac{\partial Q(\rho)}{\partial \rho}\right)^{T}\right) \\
& =\frac{\partial Q^{T}(\rho)}{\partial \rho} \Lambda Q(\rho)
\end{aligned}
$$

and the approximated Hessian of Eq. (23) can be rewritten as follows:

$$
H \approx \frac{\partial Q^{T}(\rho)}{\partial \rho} \Lambda \frac{\partial Q(\rho)}{\partial \rho}
$$

By replacing Eq. (25) and (26) in (9) the iterative solution becomes:

$$
\begin{aligned}
\rho_{i+1} & =\rho_{i}-\gamma_{i} H^{-1} J^{\prime}\left(\rho_{i}\right) \\
& =\rho_{i}-\gamma_{i}\left(\frac{\partial Q^{T}(\rho)}{\partial \rho} \Lambda \frac{\partial Q(\rho)}{\partial \rho}\right)^{-1} \frac{\partial Q^{T}(\rho)}{\partial \rho} \Lambda Q(\rho) \\
& =\rho_{i}-\gamma_{i}\left(\frac{\partial Q(\rho)}{\partial \rho}\right)^{-1} Q(\rho)
\end{aligned}
$$

Eq. (27) is known as being the iterative solution of the Newton-Raphson's method for solving the equations system $Q(\rho)=0$. Thus if the dimension $n_{\rho}$ of the controller parameters vector $\rho$ is equal to the number of specifications, the iterative algorithm of Eq. (9) is equal to that of Eq. (27) where $Q(\rho)$ is a vector and its elements are the differences between measured and desired properties in the criterion. In particular the values of the weighting factors $\lambda_{i}$ do not have any influence on the results. Moreover, the algorithm is easier to implement and the derivatives of $Q(\rho)$ with respect to $\rho$ can be computed without any additional information as follows:

$$
\frac{\partial Q(\rho)}{\partial \rho}=\left(\begin{array}{c}
\frac{\partial \omega_{c}^{T}}{\partial \rho} \\
\Phi_{m}^{\prime T} \\
K_{u}^{\prime T}
\end{array}\right)
$$

\section{Simulation Examples}

Now two different plant models are considered to show the effectiveness of the proposed method:

$$
\begin{aligned}
G_{p 1}(s) & =\frac{e^{-0.3 s}}{\left(s^{2}+2 s+3\right)^{3}(s+3)} \\
G_{p 2}(s) & =\frac{1-1.7 s}{(s+1)^{2}} e^{-0.1 s}
\end{aligned}
$$

For each plant model an initial PID controller $C_{0}$ is designed using the Kappa-Tau tuning method proposed in [3]. The proposed iterative method is then used to adjust the robustness margins and crossover frequency to the desired values and thus to impove the performance and/or the robustness and stability of the closed-loop system. Since it is typical to include a noise filter for the derivative term in the 
TABLE I

SIMULATION RESULTS

\begin{tabular}{|c|c|c|}
\hline \multirow{2}{*}{$G(s)$} & $C_{0}:\left[K_{p}, T_{i}, T_{d}\right]$ & $C_{f}:\left[K_{p}, T_{i}, T_{d}\right]$ \\
\cline { 2 - 3 } & $P_{0}:\left[\Phi_{m}, \omega_{c}, K_{u}\right]$ & $P_{f}:\left[\Phi_{m}, \omega_{c}, K_{u}\right]$ \\
\hline \hline \multirow{2}{*}{$G_{p 1}$} & {$[4.5,0.41,0.033]$} & {$[7.59,0.407,0.892]$} \\
\cline { 2 - 3 } & {$[72.3,0.136,0.233]$} & {$[60,0.23,0.331]$} \\
\hline \hline \multirow{2}{*}{$G_{p 2}$} & {$[0.127,0.647,0.10]$} & {$[0.315,1.44,0.277]$} \\
\cline { 2 - 3 } & {$[54.3,0.20,0.357]$} & {$[59.8,0.231,0.329]$} \\
\hline
\end{tabular}

PID controller, the following controller structure is used in this section:

$$
C(s)=K_{p}\left(1+\frac{1}{T_{i} s}+\frac{T_{d} s}{T_{d} / 20 s+1}\right)
$$

The results are given in the form of tables and graphs. For each model $G(s)$, the parameters of the initial controller $C_{0}=\left[K_{p}, T_{i}, T_{d}\right]$ and the related performances $P_{0}=$ $\left[\Phi_{m}, \omega_{c}, K_{u}\right]$ are given in Table I. The specifications are set to $60^{\circ}$ for the phase margin, 3 for the gain margin $\left(\frac{1}{3}\right.$ for $\left.K_{u}\right)$ and $0.23 \mathrm{rad} / \mathrm{s}$ for the crossover frequency. The parameters of the controller obtained by the iterative method are given by $C_{f}$ and the obtained performances with the new controller by $P_{f}$. It can be seen that the proposed method performs well: In only two iterations the controller that minimizes effectively the frequency criterion is obtained for each model and thus the closed-loop system has exactly the specified robustness, stability and performances. For each iteration only one identification procedure is needed. Moreover if desired, the obtained performances can be measured at the end of the controller tuning procedure with another nonparametric identification. Step responses of each simulation model with the initial and the proposed controller are shown in Fig. 3. The proposed controllers improve considerably
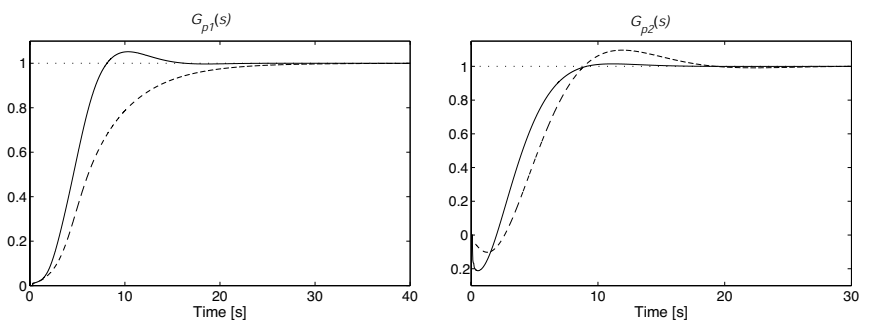

Fig. 3. Step responses (dashed line: Kappa-Tau, solid line: proposed)

the performance of the systems (the settling time is much smaller) while the robustness and stability remain as good as desired.

\section{CONCLUSION}

An iterative method for tuning the controller parameters with specifications on gain margin, phase margin and crossover frequency was proposed. The approach does not require a parametric model or any a priori knowledges about the plant. The only requirement is that a stabilizing controller exits prior to the tuning procedure. A non-parametric model of the open-loop system is then obtained at each iteration with a relay feedback test. The gain margin, phase margin, and crossover frequency as well as the gradient and Hessian of the frequency criterion are estimated directly with good accuracy using the non-parametric model. Since no assumption is made on the plant, the proposed method is neither restricted to a particular class of processes nor to a given controller structure. Simulation results show that the tuning method converges effectively to the minimum of the criterion in few iterations and can thus be used for auto-tuning of industrial plants.

\section{ACKNOWLEDGMENTS}

This research work is financially supported by the Swiss National Science Foundation under grant No. 2100064931.01

\section{REFERENCES}

[1] W. K. Ho, C. C. Hang, and L. S. Cao. Tuning of PID controllers based on gain and phase margin specifications. Automatica, 31(3):497-502, 1995.

[2] K. J. Aström and T. Hägglund. Automatic tuning of simple regulators with specifications on phase and amplitude margins. Automatica, 20(5):645-651, 1984.

[3] K. J. Aström and T. Hägglund. PID Controllers: Theory, Design and Tuning. Instrument Society of America, 2nd edition, 1995.

[4] A. Karimi, D. Garcia, and R. Longchamp. PID controller tuning using bode's integrals. Transaction on Control Systems Technology, accepted for publication.

[5] A. Karimi, D. Garcia, and R. Longchamp. Iterative controller tuning using Bode's integrals. In $C D C$, FrM07-6, pages 4227-4232, December 2002.

[6] L. Ljung. System Identification: Theory for the User. Prentice Hall, Upper Saddle River, NJ, 2nd edition edition, 1999.

[7] R. Longchamp and Y. Piguet. Closed-loop estimation of robustness margins by the relay method. IEEE ACC, pages 2687-2691, 1995.

[8] Q. Bi, Q. G. Wang, and C. C. Hang. Relay-based estimation of multiple points on process frequency response. Automatica, 33(9):1753-1757, September 1997.

[9] Q. G. Wang, T. H. Lee, H. W. Fung, Q. Bi, and Y. Zhang. PID tuning for improved performance. IEEE Transactions on CST, 7(4):3984-3989, 1999.

[10] T. S. Schei. Closed-loop tuning of PID controllers. In ACC, FA12, pages 2971-2975, 1992. 